\title{
Chromosomal bar codes produced by multicolor fluorescence in situ hybridization with multiple YAC clones and whole chromosome painting probes
}

\author{
Christoph Lengauer ${ }^{+}$, Michael R.Speicher, Susanne Popp, Anna Jauch, Masafumi Taniwaki ${ }^{\S}$, \\ Ramaiah Nagaraja', Harold C.Riethman², Helen Donis-Keller ${ }^{3}$, Michele D'Urso ${ }^{4}$, \\ David Schlessinger ${ }^{1}$ and Thomas Cremer *
}

Institut für Humangenetik und Anthropologie, Im Neuenheimer Feld 328, D-6900 Heidelberg, Germany, 'Washington University School of Medicine, Department of Molecular Microbiology, 4566 Scott Avenue, Saint Louis, MO 63110, ${ }^{2}$ The Wistar Institute, 3601 Spruce Street, Philadelphia, PA 19104, 3Washington University School of Medicine, Department of Genetics, 660 S. Euclid, Saint Louis, MO 63110, USA and ${ }^{4}$ Istituto Internationale di Genetica e Biofisica, Via Marconi 10, 80125 Naples, Italy

Received January 25, 1993; Accepted February 19, 1993

\begin{abstract}
Colored chromosome staining patterns, termed chromosomal 'bar codes' (CBCs), were obtained on human chromosomes by fluorescence in situ hybridization (FISH) with pools of Alu-PCR products from YAC clones containing human DNA inserts ranging from $100 \mathrm{kbp}$ to $1 \mathrm{Mbp}$. In contrast to conventional G- or R-bands, the chromosomal position, extent, individual color and relative signal intensity of each 'bar' could be modified depending on probe selection and labeling procedures. Alu-PCR amplification products were generated from 31 YAC clones which mapped to 37 different chromosome bands. For multiple color FISH, AluPCR amplification products from various clones were either biotinylated or labeled with digoxigenin. Probes from up to twenty YAC clones were used simultaneously to produce CBCs on selected human chromosomes. Evaluation using a cooled CCD camera and digital image analysis confirmed the high reproducibility of the bars from one metaphase spread to another. Combinatorial FISH with mixtures of whole chromosome paint probes was applied to paint seven chromosomes simultaneously in different colors along with a set of YAC clones which map to these chromosomes. We discuss the potential to construct analytical chromosomal bar codes adapted to particular needs of cytogenetic investigations and automated image analysis.
\end{abstract}

\section{INTRODUCTION}

The introduction of chromosome banding techniques in 1968 has marked a turning-point in the development and diagnostic application of cytogenetics (1). Since then improved protocols have made it possible to discriminate at least a thousand bands in human chromosome complements (2). In spite of their great importance for the assessment of chromosome structure, chromosome banding techniques have suffered from several limitations. Firstly, detailed chromosome banding analyses can only be performed using high quality chromosome spreads which are often not available for clinical and tumor cytogenetics. Secondly, the limit of resolution for the detection of deletions and the definition of breakpoints is several megabases at best. Thirdly, in spite of a steadily increasing demand for cytogenetic analyses the possibilities for automatization have remained limited by idiosyncrasies in the number, size and localization of the Gand R-bands (for review see 3 ).

During recent years, fluorescence in situ hybridization (FISH) with chromosome specific DNA probes $(4-13)$ has found widespread applications in clinical and tumor cytogenetics. Numerous examples of highly focused chromosome analyses by FISH have been published, ranging from the analysis of tumor specific translocations with probes spanning or flanking breakpoints of interest $(7-12)$ to the analysis of specific intragenic deletions (13); for a review see Lichter et al. (14). These developments have prompted molecular cytogeneticists in numerous laboratories to consider the possibility of a more specific and flexible way of chromosome banding based on FISH with nested sets of chromosome specific DNA probes (e.g. $15,16,17$, J.Gray, D.Pinkel, personal communications). Within such a scenario it should become possible to choose the localization, extension and color of each painted chromosome segment at will. We have suggested the term 'bar' to distinguish such a chromosome subregion clearly from natural chromosome bands (18). In metaphase chromosomes each bar is represented by two signals localized at corresponding sites of the two chromatids. Any combination of bars can be used to mark chromosomes at multiple, selected sites and thus produce a chromosomal bar code (CBC). For wide spread applications a large number of probes would be required covering the whole chromosome complement. We do not propose, however, that $\mathrm{CBCs}$ with hundreds of hars should be developed as an alternative

* To whom correspondence should be addressed

Present addresses: + Research Institute for Molecular Pathology, Dr. Bohr Gasse 7, A-1030 Vienna, Austria and 3rd Department of Internal Medicine, Kyoto Prefectural University of Medicine, Kamigyo-ku, Kyoto 602, Japan 
Human Molecular Genetics, 1993, Vol. 2, No. 5
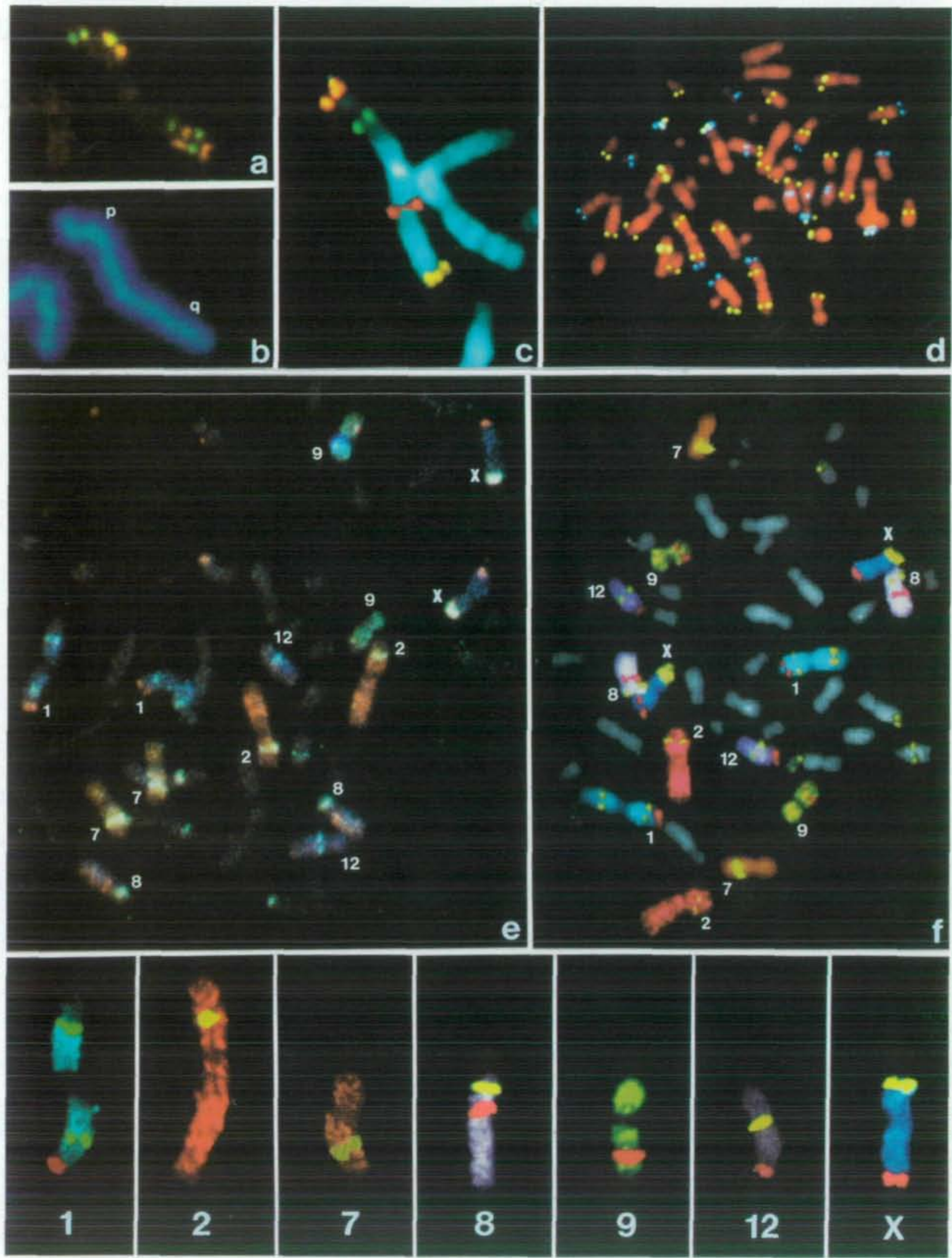
to $\mathrm{G}$ - and R-chromosome banding. Instead, we anticipate a multitude of different CBCs each consisting of a rather limited number of bars ( $<50$ per haploid chromosome set) as tools to solve specific diagnostic tasks in clinical and tumor cytogenetics (see Discussion).

The realization of $\mathrm{CBCs}$, has become possible by the generation of useful probes, the development of multicolor fluorescence in situ hybridization in order to discriminate bars by color, as well as advances in fluorescence microscopy and digital image analysis. In 1990 Nederlof et al. introduced the concept of combinatorial FISH to discriminate up to seven chromosome targets by color using three spectrally separable fluorochromes (17). These authors realized the simultaneous visualization of four chromosome-specific repetitive DNA probes with different colors. More recently, Ried et al. have applied combinatorial FISH and digital fluorescence microscopy for the multicolor painting of six chromosomes (19), while Dauwerse et al. achieved the simultaneous visualisation of twelve different chromosome pairs using chromosome specific DNA-libraries labeled with different ratios of three haptens (20).

Lichter et al. (16) and Ried et al. (19) first demonstrated the use of multiplex cosmid probes to produce colored CBCs along individual chromosomes. However, using such multiplex cosmid probes it has been difficult to achieve a hybridization efficiency satisfactory for routine, diagnostic applications of CBCs. Considering a hybridization efficiency of $70-80 \%$ to the expected target by an average cosmid clone, a series of 10 clones should yield complete hybridization patterns in only $10 \%$ or less of the hybridized metaphase spreads. For example, using six cosmid clones derived from human chromosome 5 , all 24 signals expected on the four chromatids of the two homologs (i.e. four signals for each cosmid) were only seen in some $10 \%$ of the metaphase spreads (T.Ried, personal communication). In order to implement $\mathrm{CBCs}$ as a general diagnostic tool with much larger numbers of probes, improved hybridization efficiencies are essential. For example, a multiplex probe derived from 10 clones, each with a hybridization efficiency of $99 \%$, should yield complete hybridizations in some $90 \%$ of the spreads.

Because of their large insert size, we have considered yeast artificial chromosomes (YACs) as potential candidates for the construction of CBCs. Alu-PCR can be used to selectively amplify human sequences from genomic YAC clone DNA (21).
Using such probes we have achieved hybridization efficiencies close to $100 \%$ in metaphase spreads and $>90 \%$ in interphase nuclei $(12,21)$. In this paper we demonstrate that efficient CBCs can be produced using multicolor FISH with Alu-PCR products from multiple YAC clones and whole chromosome painting probes generated from flow sorted chromosomes.

\section{RESULTS}

\section{Generation of CBCs for individual human chromosomes}

Fluorescent $\mathrm{CBCs}$ for human chromosomes $\mathrm{X}$ and 3 are exemplified in Fig. 1a and c. From eight YAC clones previously mapped to bands on the long and the short arm of the human $\mathrm{X}$ chromosome (Table 1a) Alu-PCR amplification products were labeled with either biotin or digoxigenin and used as a multiplex probe to generate a two-color bar code of alternating green and red signals along the $X$ chromosome. Fig. 1a shows a microphotograph recorded with an epifluorescence microscope by double exposure of a color film. Note that the $\mathrm{Xp}$ and $\mathrm{Xq}$ arms can be easily discriminated by the spatial ordering of the signals.

For chromosome 3, Alu-PCR amplification products from four YAC clones (Table $1 \mathrm{~b}$ ) were combined using different ratios of biotin and digoxigenin for the labeling of each clone. Although only two fluorochromes, FITC and TRITC, were used for signal detection, FITC/TRITC ratios yielded clearly distinguishable true colors of the four resulting bars seen by direct microscopic observation with a FITC/TRITC double band pass filter. Quantitation of fluorescent ratios with a cooled charged coupled device (CCD) camera and assignments of pseudocolors (Fig. 1c) were performed as described by du Manoir et al. (22).

\section{Generation of CBCs for human chromosome subsets}

To test whether a two color CBC could be constructed with 20 YACs for a subset of the human chromosome complement, AluPCR products from 11 clones were biotinylated and products from 9 others were labeled with digoxigenin. The resulting multiplex probe was used for two-color FISH to male human metaphase spreads $(46, X Y)$. Most YACs mapped to a single chromosome band, while a few, presumably chimeric YACs, marked several chromosomal bands (Table 1c). Forty-four bars were expected on the 44 autosomes, 2 bars on the X-chromosome

Figure 1. (a-d) Fluorescent chromosomal bar codes for individual human chromosomes $X$ and 3 (a,c) and human chromosome subset 1-3, 5-9, 11-13, 15-18, 22, X, Y (d). (a) Partial human metaphase spread after two-color fluorescence in situ hybridization (FISH) with a multiplex probe constructed with Alu-PCR amplified products from eight X-specific YAC clones (for clone designation, labeling and detection schemes see Table la). Alternative green and red signals can be seen along a prometaphase human X chromosome on Xp22 (green), Xp21.1 (red), Xp11.4 (green) and Xp11.3 (red), Xq24 (green), Xq25 (red), Xq26 (green) and Xq27 (red). The microphotograph was obtained by double exposure of Agfachrom $1000 \mathrm{RS}$ color slide film using a conventional fluorescence microscope. Signals on both chromatids are seen at Xp22, Xq25, Xq26 and Xq27. One signal is clearly missing at Xq24 and probably at Xp21.1. The size of the signals observed at Xp11.4 and Xp11.3 suggests that FISH was successful on both chromatids but yielded confluent signals. (b) DAPI staining of the partial metaphase spread shown in (a). (c) Partial human metapase spread after FISH with a multiplex probe consisting of Alu-PCR products from four chromosome 3 specific YAC clones (for clone designation, labeling and detection schemes see Table 1b). Alu-PCR products were labeled with different ratios of biotin and digoxigenin (see Table 1b). A cooled CCD camera was used for quantification of the resulting differences in the ratios of FITC and TRITC at each chromosomal target site. The microphotograph was recorded from a color screen. YAC clone yWPR409 (localized at 3p25) is shown in orange, HIG6 (3p21) in green, HIF8 (3q21) in red and HIH8 (3q28) in yellow. False colors reflect significant differences of the recorded fluorescent ratios. These differences were already apparent by conventional fluorescence microscopy (data not shown). (d) Male human metaphase spread $(46, X Y)$ after two-color FISH with a multiplex probe constructed with biotinylated Alu-PCR products from 11 YAC clones (detected with FITC) and digoxigenin labeled Alu-PCR products from 9 additional clones (detected with TRITC) (for details of the clones see Table 1c). Chromosomes were counterstained with DAPI. The signals were recorded with a CCD camera, processed using digital image analysis and presented in false colors (DAPI: red; FITC: yellow; TRTC: blue). All 47 bars expected from this mulplex probe (see text) can be seen. (e,f) Two female metaphase spreads (46, XX) after simultaneous combinatorial fluorescence in sim hybridization of directly fluorochromated whole chromosome painting (WCP) libraries for chromosome 1,2,7,8,9,12 and the human X chromosome as well as Alu-PCR amplified proxtucts of 10 YAC clones labeled either with biotin or digoxigenin (for clone designation, labeling and detection schemes see text and Table lc). YAC clones were selected to provide additional bars on painted metaphase chromosomes in distinctly differemt colors. Two clones provided additional signals on non-painted chromosomes 6 and 13. e) Microphotograph obtained by triple exposure of color slide film using conventional fluorescence microscopy. n Pseudocolored digital imsge recorded with the CCD camera (for details see Methodology). Lower row: Enlarged pseudocolored, painted and bar-ooded chromosomes $1,2,7,8,9,12$ and $X$. 
and 1 bar on the Y-chromosome. Hybridization signals were recorded using a CCD camera and processed with digital image analysis. A typical metaphase spread with the resulting two-color bar code is demonstrated in Fig. 1d. Fig. 2 shows the G-banded karyotype and the CBC established by this model experiment. Note that the localization, color and intensity of the bars allow the unequivocal identification of the 18 labeled chromosomes.

Table 2 lists the reproducibility of each bar in 15 metaphase spreads which were G-banded and photographed prior to FISH. For twelve bars the signals were observed in all evaluated metaphase spreads at the respective target band of both homologues and on both chromatids. Successful hybridization to both chromatids was also assumed when single, symmetrical signals suggested that two signals had become confluent. Signal counting was considerably facilitated by digital image analysis with optimized signal thresholding. Seven of the 15 evaluated metaphase spreads exhibited all signals (i.e. 94 signals for the 47 bars), while all spreads exhibited at least $78 \%$ of all expected signals. The relative intensity of the signals also appeared highly reproducible. For example the signal on $7 \mathrm{q} 31$ which was generated by FISH of Alu-PCR amplification products of the 790 kb YAC yCF-1/7/5-R established from the cystic fibrosis region (23) clearly gave the most intense signals. Signals of somewhat lower intensities were produced on the pseudoautosomal region of the $\mathrm{X}$ and $\mathrm{Y}$ chromosomes by Alu-PCR products of the $1 \mathrm{Mbp}$ sized YAC clone MIC2. The latter clone reproducibly showed another, considerably weaker signal on 8p21-22.

We have also studied the feasibility of combining multiple-color FISH with fluorochrome-labeled whole chromosome painting (WCP) probes and and multiple YAC clones. Seven chromosome types were painted in different colors, including human chromosomes \# 1 (labeled with Spectrum Green and coumarin-4-dUTP), \#2 (Spectrum Orange only), \# 7 (Spectrum Green and Spectrum Orange), \# 8 (Spectrum Green, Spectrum Orange and coumarin-4-dUTP), \# 9 (Spectrum Green only), \# 12 (Spectrum Orange and coumarin-4-dUTP) and $\mathrm{X}$ (coumarin-4-dUTP only). Alu-PCR amplification products from 10 YAC clones were labeled either with biotin (clones EG8, YAC35, yCF-1/7/5-R, MIC2, EO2155; FITC detection) or digoxigenin (clones HTY3153, HTY3195, HTY3137, yWXD526, yWXD534; TRITC detection) and added to the hybridization mixture to create a two color CBC (green and red) on the painted human chromosome subset. Fig. le,f shows two metaphase spreads with painted and bar coded chromosomes. For comparison, Fig. le shows a real color microphotograph obtained by triple exposed color slide film, while Fig. If presents a digitized and pseudocolored image.

\section{DISCUSSION}

In the present study Alu-PCR amplified products from multiple human YAC clones were combined to yield chromosomal bar codes (CBCs) for individual chromosomes and chromosomal subsets with up to four colors. In some experiments, a CBC derived with a multiplex YAC-probe was combined with multiplecolor whole chromosome painting. Signal reproducibilities for CBCs were highly superior to previous experiments with multiplex cosmid probes. In the following discussion we will consider firstly technical aspects of the development of CBCs and secondly the potential usefulness of this approach within the framework of classical and molecular cytogenetics.

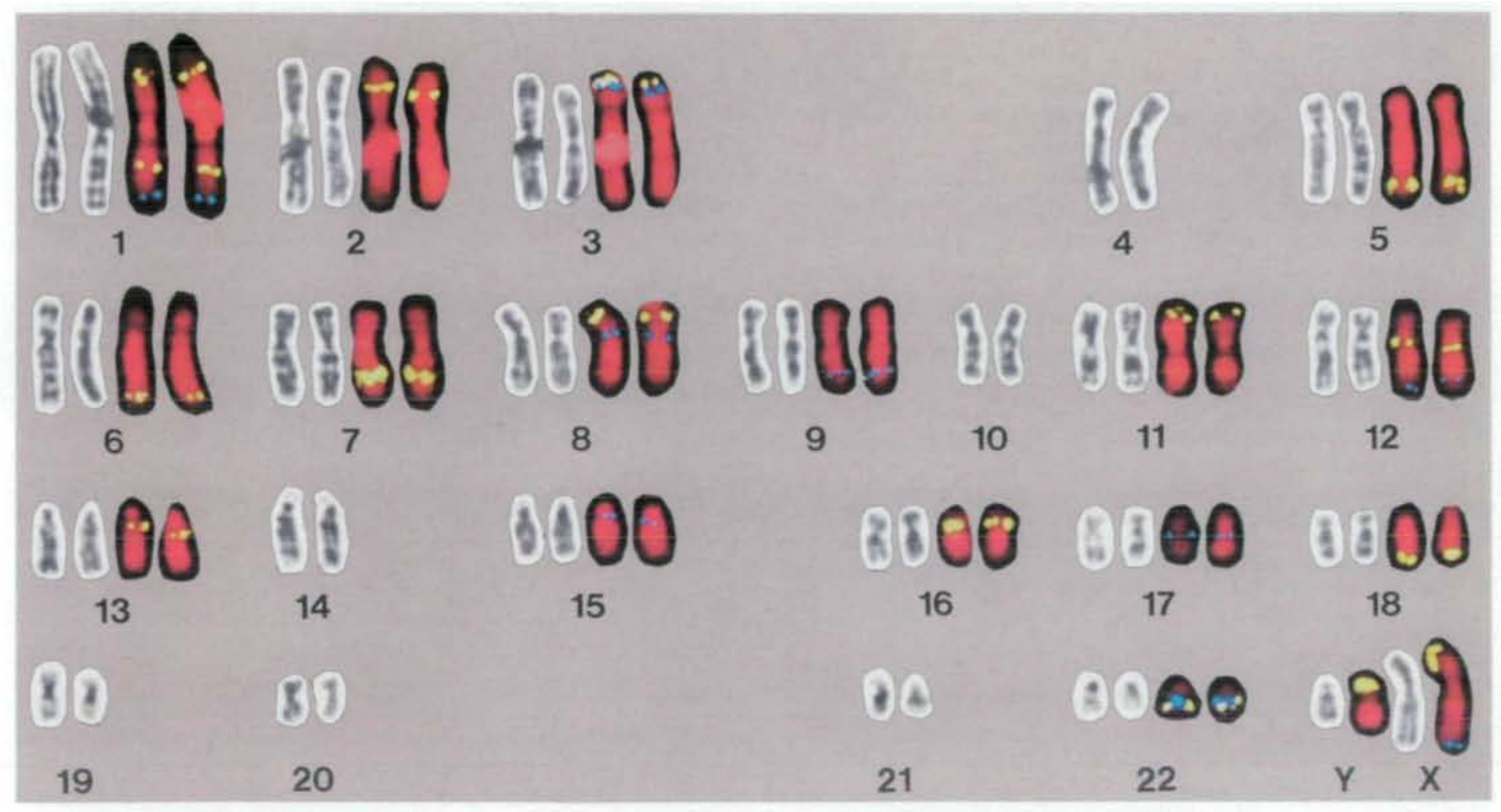

Figure 2. Karyotype $(46, X Y)$ established from a metaphase spread with GTG-banded (left) and bar coded chromosomes (right). FISH was performed following GTG-banding with the multiplex probe described in Table 1c. All 47 bars expected from this multplex probe can be detected on the digitized chromosomes (for details compare Fig. 1d). 


\section{Development of multiple-color bar codes}

Multiple-color FISH based on probes labeled with different haptens requires complex detection schemes. This difficulty can be avoided by the use of probes directly labeled with fluorochromes as shown in the present paper for multiple-color FISH with WCP-probes. Fluorochrome labeled probes detecting DNA targets of a few kbp have been used successfully for FISH (24) and the number of spectrally separable fluorochromes with high quantum yields will likely increase considerably in the near future. As a result, it can be anticipated that multiple color FISH should become a routine procedure in cytogenetic laboratories. While microphotographs showing 'real color pictures' (25) can be taken directly on color slides as demonstrated by Dauwersee et al. (20), approaches for the quantitative evaluation of multicolor FISH by digital fluorescence microscopy and image analysis have also been developed $(22,26)$ and will become indispensable for the automated evaluation of CBCs (see below).

A number of Alu-PCR amplified YACs used for the present experiments yielded chromosomal bars on target chromosome bands of both homologs and both chromatids in all evaluated metaphase spreads. The hybridization efficiency of other YACs ranged between 78 and $98 \%$ and depends on the number and distance of the Alu elements, as well as the primers and amplification conditions used (21). In general it can be expected that large clones from Alu-rich G-light bands of the human genome should yield optimal results, although we have successfully mapped numerous Alu-PCR amplified YACs to Gdark bands as well $(12,21$, and our unpublished data). Alternatively, for YAC clones from Alu-poor regions of the genome, purification of the YACs by pulsed field gel electrophoresis can be combined with universal DNA

Table 1. Origin, designation, localization and labeling scheme of YAC clones used for the construction of bar codes for individual human chromosomes (a,b) and human chromosome subset 1-3, 5-9, 11-13, 15-18, 22, X, Y (c) In some cases clones have been redesignated. Designations from the cited publications are given in parenthesis. Biotinylated probes were detected with FITC, probes labeled with digoxigenin were detected with TRITC.

\begin{tabular}{|c|c|c|c|}
\hline YAC clone & localization & labeling & source \\
\hline \multicolumn{4}{|c|}{ (a) $X$ chromosome bar code } \\
\hline yWXD978 & $\mathrm{Xp} 22$ & biotin & Ref. 44 \\
\hline yWXD1017 & Xp21.1 & digoxigenin & Ref. 44 \\
\hline yWXD975 & Xp11.4 & biotin & Ref. 44 \\
\hline yWXD967 & Xpl 1.3 & digoxigenin & Ref. 44 \\
\hline yWXD432 & $X q 24$ & biotin & Ref. 45 \\
\hline yWXD402 & $\mathrm{Xq} 25$ & digoxigenin & Ref. 45 \\
\hline yWXD382 & $\mathrm{Xq26}$ & biotin & Ref. 45 \\
\hline yWXD393 & $\mathrm{Xq} 27$ & digoxigenin & Ref. 45 \\
\hline \multicolumn{4}{|c|}{ (b) chromosome 3 bar code } \\
\hline yWPR409 (A168H4) & $3 p 25$ & $1 \times$ biotin $/ 3 \times$ digoxigenin & Ref. 12 \\
\hline HIG6 & $3 \mathrm{p} 21$ & biotin & (a) \\
\hline HIF8 & $3 q 21$ & digoxigenin & (a) \\
\hline HIH8 & $3 q 28$ & $3 \times$ biotin $1 \times$ digoxigenin & (a) \\
\hline \multicolumn{4}{|c|}{ (c) bar code for human chromosome subset } \\
\hline EG8 & $1 \mathrm{p} 32,1 \mathrm{q} 32,6 \mathrm{q} 25$ & biotin & Ref. 44 \\
\hline HTY 3153 & 1944 & digoxigenin & (b), Ref. 46 \\
\hline YAC 35 & $2 \mathrm{p} 21,13 \mathrm{q} 14$ & biotin & (c) \\
\hline yWPR409 (A168H4) & $3 \mathrm{p} 25$ & digoxigenin & Ref. 12 \\
\hline HTY 3191 & $3 p 26$ & biotin & (b), Ref. 46 \\
\hline yWPR411 (D73A7) & $5 q 33$ & biotin & Ref. 12 \\
\hline $\mathrm{yCF}-1 / 7 / 5-\mathrm{R}$ & $7 q 31$ & biotin & Ref. 23 \\
\hline $\mathrm{MIC2}$ & $\begin{array}{l}\text { Xp22.3, Yp11.3, } \\
8 \mathrm{p} 21-22\end{array}$ & biotin & (d), Ref, 47 \\
\hline HTY 3195 & $8 q 11,12 q 24$ & digoxigenin & (b), Ref, 47 \\
\hline HTY 3137 & $9 q 31-33$ & digoxigenin & (b), Ref, 47 \\
\hline$Y-11-5$ & $11 \mathrm{p} 14-15$ & biotin & (e) \\
\hline $\mathrm{EO} 2155$ & $12 q 13$ & biotin & (f) \\
\hline YAC 36 & $15 q 11$ & digoxigenin & (c) \\
\hline HTY 3150 & $16 \mathrm{p} 11$ & biotin & (b), Ref. 46 \\
\hline yWPR412 (D122A5) & $17 q 12-21.1$ & digoxigenin & Ref. 12 \\
\hline DKHR 7 & $18 q 22-23$ & biotin & (b), Ref. 46 \\
\hline yWPR415 (D107F9) & $22 \mathrm{q} 11$ & digoxigenin & Ref. 12 \\
\hline A22604 & $22 q 12$ & biotin & (g), Ref. 44 \\
\hline yWXDS26 & $\mathrm{Xq} 27-28$ & digoxigenin & Ref. 48 \\
\hline yWXD534 & $\times q 27-28$ & digoxigenin & Ref. 48 \\
\hline
\end{tabular}

(a) K.Gardiner, R.Williarns; Eleanore Roosevelt Institute, Denver, CO, USA; unpublished

(b) H.Donis-Keller, Washington Univ. School of Medicine, Dept. of Genetics, Saint Louis, MO, USA; H.C. Riathman,

The Wistar Instutute, Philadelphia, PA, USA; unpublished

(c) B.Horsthemke, Institute of Human Genetics, Essen, Germany; unpublished

(d) G.Rappold, Institute of Human Genetics, Heidelberg, Germany; unpublished

(e) J.Hoovers, Institute of Human Genetics, Amsterdam, The Netherlands; unpublished

(f) M.Ross, Imperical Cancer Research Fund, London, UK; unpublished

(g) B.Emanuel, Children's Hospital, Univ. of Pennsylvania, Philadelphia, PA, USA; unpublished 
amplification protocols $(27,28,29)$ to generate useful probes (our unpublished data). Recently YAC libraries have been constructed for the human genome with YACs containing an average insert size of $1 \mathrm{Mbp}$ (30). YAC contigs are rapidly becoming available from these libraries for every human chromosome (31). This development will provide an ideal opportunity to collect a large collection of mapped reference YACs for molecular cytogenetic purposes spaced over the whole human chromosome complement. Each probe should be ideally defined by sequence tagged sites (STS). Using standard FISH protocols each YAC or other probe included into the reference collection should yield a bar at a single chromosome band with high efficiency ( $>95 \%$ in human lymphocyte metaphase spreads and $>90 \%$ interphase nuclei). To avoid cumbersome steps of cloning and DNA preparation for cytogenetic laboratories not well equipped for such purposes, it seems preferable that all probes contained in this collection could be amplified by PCR. Based on the availability of such a collection molecular cytogeneticists could construct a multitude of specific CBCs with unprecedented flexibility. While YAC contigs could be used in order to produce more extended bars, cosmid contigs could be applied to produce localized multicolor CBCs for high resolution purposes. Each CBC could be adapted to optimally solve a specific diagnostic task. For most practical purposes a fairly limited number of 5-50 bars would be sufficient. For example, a potential telomere integrity assay for detection of cryptic translocations could be based on a multicolor $\mathrm{CBC}$ defining the telomeric regions of each individual chromosome in a different color (32). Other examples are discussed below.

\section{Applications of chromosomal bar codes}

Multiple-color $\mathrm{CBCs}$ as described above are obviously not useful to screen whole chromosome complements for unknown aberrations. In order to judge the potential diagnostic value of specific CBCs, it is important to consider their application in the context of an integrated concept of chromosome analysis (22). For a global analysis conventional chromosome banding remains the procedure of choice, wherever high quality metaphase spreads are available. A new approach, termed comparative genomic hybridization (CGH), has recently been developed $(33,22)$. CGH provides information on over- or underrepresented chromosomes or chromosome segments in the test genome even in cases where genomic DNA, prepared e. g. from a solid tumor or from tissue of a patient with a suspected chromosomal syndrome, is the only source available for analysis. Depending on the results of global assays, such as chromosome banding and/or CGH analyses, a specific CBC can be constructed to independently confirm chromosomal aberrations and analyze them in more detail. Suspected translocations, insertions, deletions and inversions may become easily recognizable by a change in the sequence of differently colored bars spaced along the chromosomes of interest. If desirable, breakpoints can be defined with localized multicolor CBCs at any desirable level of resolution. If marker chromosomes of unknown origin still remain, multiple-color FISH with sets of WCP probes can be used to deduce the chromosomes which have contributed to their formation, followed again, if desirable, by the application of a CBC tailored to the unequivocal identification of specific chromosomal subregions (34). Notably,

Table 2. Hybridization efficiency ( $\mathrm{n}=15$ metaphase spreads) of a multiplex probe constructed with AluPCR amplified products from 20 YAC clones (compare Fig. 1d,e)

\begin{tabular}{|c|c|c|}
\hline YAC clone & signal localization & $\begin{array}{l}\text { \% metaphase spreads with } \\
\text { signals on both chromatids } \\
\text { of both homologs }\end{array}$ \\
\hline
\end{tabular}

\begin{tabular}{|c|c|c|}
\hline \multicolumn{3}{|c|}{ Blotinylated probes detected with FITC } \\
\hline \multirow[t]{3}{*}{ EG8 $1 \mathrm{p} 32$} & 100 & \\
\hline & $\operatorname{lq} 32$ & 100 \\
\hline & $6 q 25$ & 90 \\
\hline \multirow{2}{*}{ YAC 35} & $2 \mathrm{p} 21$ & 100 \\
\hline & $13 q 14$ & 90 \\
\hline HTY 3191 & $3 p 26$ & 98 \\
\hline yWPR411 (D73A7) & $5 q 33$ & 100 \\
\hline YCF-1/7/5-R & $7 q 31$ & 100 \\
\hline \multirow{3}{*}{ MIC2 } & $8 p 21-22$ & 97 \\
\hline & $x p 22.3$ & $100 *$ \\
\hline & Yp11.3 & $100^{*}$ \\
\hline Y-11-5 & $11 \mathrm{p} 14-15$ & 87 \\
\hline EO2155 & $12 \mathrm{q} 13$ & 97 \\
\hline HTY 3150 & 16 pl1 & 100 \\
\hline DKHR 7 & $18922-23$ & 100 \\
\hline A22604 & $22 \mathrm{q} 12$ & 78 \\
\hline \multicolumn{3}{|c|}{ Dtgoxigenin labeled probes detected with TRTC } \\
\hline HTY 3153 & $1 q 44$ & 98 \\
\hline yWPR409 (A168H4) & $3 \mathrm{p} 25$ & 100 \\
\hline \multirow[t]{2}{*}{ HTY 3195} & $8 q 11$ & 100 \\
\hline & $12 q 24$ & 78 \\
\hline HTY 3137 & $9431-33$ & 92 \\
\hline YAC 36 & $15 q 11$ & 88 \\
\hline yWPR412 (D122A5) & $17 q 12-21.1$ & 78 \\
\hline yWPR415 (D107F9) & $22 \mathrm{~g} 11$ & 100 \\
\hline yWXD526 + yWXD534 & $x q 27-28$ & $87 *$ \\
\hline
\end{tabular}

*Only one homolog present 
chromosomal subregions (34). Notably, chromosome analyses by $\mathrm{CBCs}$ can also be performed with metaphase spreads of inferior quality not suitable for banding analyses. CBCs comprising a small number of multiple color bars could even be constructed in a way which makes them applicable for interphase cytogenetics.

Clinical and tumor cytogenetics often permits one to focus the analysis a priori on a subset of chromosomes or chromosomal subregions. For example, chromosome targets of major importance with regard to disease status, differential diagnosis and possible prognosis are clearly different in cytogenetic analyses of a child with neuroblastoma, a patient with acute nonlymphatic leukemia or in case of a woman asking for prenatal chromosome screening. Specifically constructed, multicolor CBCs will help to speed up such analyses dramatically, since they can provide comprehensive information in a single FISH experiment with regard to a set of numerical and/or structural aberrations specifically important for the case in question (35). Reprobing of DNA:DNA in situ hybridization preparations (36) should provide the possibility to apply fluorescence chromosome banding together with multiple-color chromosome painting and CBCs in sequential order, even when the number of metaphase spreads available for analysis is small which is often the case in tumor materials.

-In addition to their diagnostic specificity, $\mathrm{CBCs}$ can be adapted to the needs of automated chromosome analysis $(3,37)$. Using conventional banding, the whole chromosome complement has to be segmented and analyzed by automated evaluation procedures even in cases where the evaluation of a chromosome subset appears sufficient to obtain the required diagnostic information. In contrast, simple thresholding algorithms (38) can be applied to home in on such a subset visualized by a specific CBC. In combination with fluorescence banding bars may also serve as additional landmarks for the unequivocal discrimination of 'problem' chromosomes or chromosomal subregions that cannot easily be distinguished automatically by virtue of their banding characteristics alone.

\section{MATERIALS AND METHODS}

\section{Cell matertal}

Metaphase spreads and interphase nuclei were prepared from phytohemagglutininstimulated normal male and female human blood lymphocytes using standard techniques of colcemid treatment, hypotonic treatment and methanol/acetic acid fixation. Preperations were stored in $70 \%$ ethanol at $4^{\circ} \mathrm{C}$ until use. Chromosome spreads were GTG-banded, photographod with a black and white film (AgfaOrtho) and postrixed with $3.7 \%$ buffered formaldehyde (39). Prior to use in FISH experiments, slides were pretreated with RNAse A (Boehringer Mannheim) (100 $\mu \mathrm{g} / \mathrm{ml})$ in $2 \times \mathrm{SSC}$ for $60 \mathrm{~min}$ at $37^{\circ} \mathrm{C}$ followed by a pepsin digestion $(50 \mu \mathrm{g} / \mathrm{ml})$ in $0.01 \mathrm{M} \mathrm{HCl}$ for $10 \mathrm{~min}$ at $37^{\circ} \mathrm{C}$ and a postfixation step in $1 \%$ acid-free formaldehyde in phosphate-buffered saline/ $\mathrm{MgCl}_{2}$ for $10 \mathrm{~min}$ at room temperature as described (11).

\section{DNA probes}

Phasmid libraries from sorted human chromosomes $1,8,12$ and $X$ were a generous gift from Dr Joe Gray, University of California, San Francisco, CA (40) Amplification and DNA preparation of these libraries were carried out according to Maniatis a al. (41). WCP probes directly conjugated to a fluorochrome, i.e. Spectrum Green for chromosomes 1, 7, 8 and 9, and Spectrum Orange for chromosomes 2, 7, 8 and 12, were provided by Imagenetics (Naperville, IL). YAC clones were kindly contributed by several investigators (see Table 1) either as purified genomic DNA or in agarose-plugs prepared for pulsed field gel electrophoresis according to standard techniques (42).

\section{Ahu-PCR amplification of YAC clones and probe labeling}

For FISH of YAC clones human sequences were amplified by Alu-PCR from genomic YAC clone DNA as described in detail elsewhere (21). Alu-PCR amplification products were labeled with biocin-11-dUTP or digoxigenin-11-dUTP using standard nick translation procedures (43). In the nick translation reaction of the plasmid library DNAs from sorted human chromosomes, aminomethylcoumarin acetic acid coumarin-4-(AMCA) dUTP (a generous gift of Amersham, UK) was used at a concentration of $0.025 \mathrm{mM}$, together with unlabeled nucleotides dATP, dCTP and dGTP at a concentration of $0.16 \mathrm{mM}$ each and unlabeled dTTP at $0.008 \mathrm{mM}$.

Assembly of multiplex probes, fluorescence in situ hybridtzatton and probe detection

Multiplex probes prepared from up to $20 \mathrm{YAC}$ clones were prepared by mixing Alu-PCR products from individual YAC clones. For each clone an amount of probe was added to the mixture which yielded optimum results in a single FISH experiment (range 50-200 ng). In some experiments, multiplex YAC-probes were combined with combinations of fluorochrome conjugated Imagenetics WCPprobes, labeled with Spectrum Green or Spectrum Orange, as well as WCP-probes labeled with coumarin-4-dUTP. For each WCP-probe amounts yielding optimum signal intensities in combinatorial FISH experiments were determined empirically (2.5 to $15 \mu$ of Imagenetics WCP-probes diluted $1: 10$ in $10 \mathrm{mM}$ Tris $-\mathrm{HCl} / 1 \mathrm{mM}$ EDTA and 1-2 $\mu \mathrm{g}$ of plasmid DNA libraries labeled with coumarin-4-dUTP). Pooled probes were precipitated in the presence of $300 \mu \mathrm{g}$ Cot-1 DNA (Life Technologies, Eggenstein, Germany), $50 \mu \mathrm{g}$ sonicated salmon sperm DNA (Sigma, Munich, Germany) and $300 \mathrm{mM}$ sodium acetate. DNA was dissolved in $10 \mu$ of hybridization mixture yielding a finsl concentration of $50 \%$ deionized formamide, $1 \times$ SSC $(\mathrm{pH} 7.0)$ and $10 \%$ dextran sulphate. Multiple color FISH, posthybridization washing steps, and detection procedures for biotinylated and digoxigenin labeled probes were carried out as described (43). In experiments which included whole chromosome painting libraries and YAC probes, slides were washed $3 \times 5 \mathrm{~min}$ in $50 \%$ formamide/ $1 \times \mathrm{SSC}$ at $45^{\circ} \mathrm{C}$ and $5 \mathrm{~min}$ in $2 \times \mathrm{SSC}$ at $37^{\circ} \mathrm{C}$.

\section{Conventional and digital fluorescence microscopy}

A Zeiss Axiophot epifluorescence microscope was used for conventional flworescence microscopy. Microphotographs on triple exposed color slide films (Agfachrom 1000 RS or Kodak Ektachrome 400) were obtained using filter sets purchased from Cart Zeiss (Oberkochen, Germany) (double band pass filter set 23 for the simultaneous observation of FITC and rhodamine signals, filter set 09 (BP 450-490, FT 510, LP 515-565) for FITC signals, filter set 01 (BP 365, FT 395, LP 397) for coumarin signals). For digital fluorescence microscopy the Axiophot was coupled to a cooled CCD camera (Photometrics Kodak KAF 1400 , Tucson, AZ). A Macintosh Quadra 900 was used for camera control and digital image acquisation in the 'tag image file format' (TIFF) using the software package Nu200 2.0 (Photometrics). Three gray scale fluorescence images were recorded for each metaphase spread, using filter set 15 (BP 546, FT 580, LP 590 ) for thodamine signals in addition to filter sets 01 and 09 . After changing the format of the images from 'TIFF' to the Macintosh specific 'PICT'-format, further processing of the images, including merging and pseudocoloring, was performed with the software package 'Gene Join' as described by Ried at al. (19). Shifts between the three images were corrected using double and triple colored probe signals as reference.

\section{ACKNOWLEDGEMENTS}

Conceptual discussions with Peter Lichter (Deutsches Krebsforschungszentrum, Heidelberg, Germany) and Johannes Wienberg (Institut fur Anthropologie and Humangenetik, München, Germany) are gratefully acknowledged. We also thank Beverly Emanuel (Children's Hospital, University of Pennsylvania, Philadelphia, PA, USA), Kathleen Gardiner and Reginald Williams (Eleanore Roosevelt Instiute, Denver, CO, U.S.A.), Eric Green (Washington University School of Medicine, St Louis, MO, USA), Jan Hoovers (Institute of Human Genetics, Amsterdam, The Netherlands), Bernhard Horsthemke (Institut fur Humangenetik, Essen, Germany), Antony Monaco (Instinute of Molecular Medicine, John Radcliffe Hospital, Oxford, UK), Gudnun Rappold (Institut für Humangenetil und Anthropologie, Heidelberg, Germany), and Mark Ross (Imperial Cancer Research Fund, London, UK) for providing YAC clones. The Carl Zeiss Company (Oberkochen, Germany) has kindly supporred the print of the color figures. This work was supported by grants from the European Community (GENOCT91-0029), the Deutsche Forschungsgemeinschaft (Cr 59/14-1) and the NIH (HG00567, HR).

\section{ABBREVIATIONS}

CBC: chromosomal bar code CCD: charge coupled device DAPI: 4',6'-diamidino-2-phenylindole 
dATP: desoxyadeninetriphosphate dCTP: desoxycytosinetriphosphate dGTP: desoxyguaninetriphosphate dTTP: desoxythymidinetriphosphate dUTP: desoxyuridinetriphosphate FISH: fluorescence in situ hybridization FITC: fluorescein isothiocyanate PCR: polymerase chain reaction TIFF: tag image file format TRITC: tetramethyl thodamine isothiocyanate WCP: whole chromosome painting YAC: Yeast arificial chromosome

\section{REFERENCES}

1. Caspersson, T., de la Chapelle, S., Foley, G.E., Kudynowski, J., Modest, E.J., Simonsson, E., Wagh, V. and Zoch, L. (1968) Joumal Exp. Cell Res. 49, 219-226.

2. Yunis, J.J. (1976) Science 191, $1268-1270$.

3. Rutovitz, D. (1992) J. Radiar. Res. 33, Suppl., 1-30.

4. Cremer, T., Lichter, P., Borden, J., Ward, D.C. and Manuelidis L. (1988) Hum. Genet. 80, 235-246.

5. Lichter, P., Cremer, T., Borden, J., Manuelidis, L. and Ward, D.C. (1988) Hum. Gener. 80, 224-234.

6. Pinkel, D., Landegent, J., Collins, C., Fuscoe, J., Segraves, R., Lucas, J., and Gray, J. (1988) Proc. Natl. Acad. Sci. U.S.A. 85, 9138-9142.

7. Rowley, J.D., Diaz, M.O., Espinosa, R., Patel, J.D., van Melle, E., Ziemin, S., Taillon-Miller, P., Lichter, P., Evans, G.A., Kersey, J.H. and Ward, D.C. (1990) Proc. Natl. Acad. Sci. U.S.A. 87, 9358-9362.

8. Goa, J., Erickson, P., Gardiner, K., Le Beau, M.M., Diaz, M.O., Patterson, D., Rowley, J.D. and Drabkin, H.A. (1991) Prac. Natl. Acad. Sci. U.S.A. $88,4882-4886$.

9. Selleri, L., Hermanson, G.G., Eubanks, J.H., Lewis, K.A., Evans, G.A. (1991) Proc. Natl. Acad. Sci. U.S.A. 88, 887-891.

10. Heitz, D., Rousseau, F., Devys, D., Saccone, S., Abderrahim, H., Le Paslier, D., Cohen, D., Vincent, A., Tonido, D., Della Valle, G., Johnson, S., Schlessinger, D., Oberle, I. and Mandel, J.L. (1991) Science 251, $1236-1239$

11. Ried, T., Lengauer, C., Cremer, T., Wiegant, J., Raap, A.K., van der Ploeg, M., Groitl, P. and Lipp, M. (1992) Genes, Chrom. \& Cancer 4, 69-74.

12. Lengauer, C., Riethman, H.C., Speicher, M.R., Taniwaki, M., Konecki, D., Green, E.D., Becher, R., Olson, M.V. and Cremer, T. (1992) Cancer Res. 52, 2590-2596.

13. Ried, T., Mahler, V., Vogt, P., Blonden, L., van Ommen, G.J.B., Cremer, T. and Cremer, M. (1990) Hum. Genet. 85, 581-586.

14. Lichter, P., Boyle, A.L., Cremer, T. and Ward, D.C. (1991) Genet. Anal. Tech. Appl. 8, 24-35

15. Cremer, T., Landegent, J, Brückner, A., Scholl, H.D., Schardin, M., Hager, H.D., Devilee, P., Pearson, P. and van der Ploeg, M. (1986) Hum. Genet. $74,346-352$

16. Lichter, P., Tang, C.J.C., Call, K., Hermanson, G., Evans, G.A., Housman, D. and Ward, D.,C. (1990) Science 247, 64-69

17. Nederlof, P.M., van der Flier S., Wiegant, J., Raap, A.K., Tanke, H.J., Ploem, J.S. and van der Ploeg, M. (1990) Cyrometry 11, 126-131

18. Cremer T., Lengauer C., Popp S., Scherthan, H. and Wienberg, J. (1991) Am. J. Hum. Genet. Supplement 49 Abstract No. 189

19. Ried, T., Baldini, A., Rand, T.C. and Ward, D.C. (1992) Proc. Natl. Acad. Sci. U.S.A. 89, $1388-1392$.

20. Dauwerse, J.G., Wiegant, J., Raap, A.K., Brauning, M.H. and van Ommen, G.J.B. (1992) Hum. Molec. Gener. 1, 593-598.

21. Lengauer, C., Green, E.D. and Cremer, T. (1992) Genomics 13, 286-289.

22. du Manoir, S., Speicher, M.R., Joos, S., Schröck, E., Popp, S., Döhner, H., Kovacs, G., Robert-Nicoud, M., Lichter, P. and Cremer, T. (1993) Hum. Genet. 90, 590-610.

23. Green, E.D. and Olson, M.V. (1990) Science 250, 94-98.

24. Wiegant J., Ried, T., Nederlof, P.M., van der Ploeg, M., Tanke, H.J. and Raap, A.K. (1991) Nucleic Acid Res 19, 3237-3241

25. Lodbetter, D.H. (1992) Hum. Molec. Genet. 1, 297-299.

26. Nederlof, P.M., van der Flier, S., Vrolijk, J., Tanke, H.J. and Raap, A.K. (1992) Cytometry 13, 839-845.

27. Melzzer, P.S., Guan X.Y., Burgess, A. and Trent, J.M. (1992) Nature Genetics 1, 24-28.

28. Telenius, H., Pelmear, A.H., Tunnacliffe, A., Carter, N.P., Behmel, A., Ferguson-Smith, M.A., Nordenskjöld, M., Pfragner, R. and Ponder, B.A.J. (1992) Genes, Chrom \& Cancer 4, 257-263.
29. Zhang, L., Cui, X., Schmitt, K., Hubert, R., Navidi, W. and Amheim, N. (1992) Proc. Natl. Acad. Sci. U.S.A. 89, 5847-5851.

30. Bellanne-Chantelot, C., Lacroix, B., Ougen, P., Billault, A., Beaufils, S., Bertrand, S., Georges, I., Glibert, F., Gros, I., Lucotte, G., Susini, L., Codani, J-J., Gesnouin, P., Pook, S., Vaysseix, G., Lu-Kuo, J., Ried, T., Ward, D.C., Chumakov, I., Le Paslier, D., Barillor, E., and Cohen, D.(1992) Cell 70, $1059-1068$.

31. Chumakov, I., Rigault, P., Guillou, S., Ougen, P., Billaut, A., Guasconi, G., Gervy, P., LeGall, I., Soularue, P., Grinas, L., Bougueleret, L., Bellanne-Chantelot, C., Lacroix, B., Barillot, E., Gesnouin, P., Pook, S., Vaysseix, G., Frelat, G., Schmitz, A., Sambucy, J.-L., Bosch, A., Estivill, X., Weissenbach, J., Vignal, A., Riethman, H., Cox, D., Patterson, D., Gardiner, K., Hattori, M., Sakaki, Y., Ichikawa, H., Ohki, M., Le Paslier, D., Heilig, H., Antonarakis, S., and Cohen, D. (1992) Nature 359, 380-387

32. Lodbetter, D.H. (1992) Am. J. Hum. Genet. 51, 451-456

33. Kallioniemi, A., Kallioniemi, O.P., Sudar, D., Rutovitz, D., Gray, J.W., Waldman, F. and Pinkel, D. (1992) Science 258, 818-821

34. Popp, S., Jauch A., Schindler, D, Speicher, M.R., Lengauer, C., DonisKeller, H., Riethman, H.C. and Cremer, T. (1993) Hum. Genet. In Press.

35. Ried, T., Landes, G., Dackowski, W., Klinger, K. and Ward, D.C. (1992) Hum. Molec. Genet. 1, 307-313

36. Heslop-Harrison, J.S., Harrison, G.E., Leitch, I.J. (1992) Trends in Genetics 81, 372-373.

37. Lundsteen, C., Piper, J. (eds). (1989) Automation of Cytogenetics. (Springer Verlag, Berlin, Heidelberg, New York).

38. Cremer, C., Remm, B., Bischoff, A., Vollweiler, T. (1992) J. Radiat. Res. 33, Suppl., 189-205.

39. Klever, M., Grond-Ginsbach, C., Scherthan, H., Schroeder-Kurth, T. (1991) Hum. Genet. 86, 484-486.

40. Collins, C.C., Kuo, W.L., Segraves, R., Fuscoe, J.C., Pinkel, D. and Gray, J.W.(1991) Genomics 11, 997-1006.

41. Maniatis, T., Fritsch, E.F. and Sambrook, J. (1982) Molecular Cloning: A Laboratory Manual. Cold Spring Harbor.

42. Burke, D.T., Carle, G.F. and Olson, M.V. (1987)Science 236, 806-812.

43. Lichter, P. \& Cremer, T. (1992) Human Cytogenetics: a practical approach (IRL-press, Oxford University Press, Oxford, New York, Tokyo).

44. Brownstein, B.H., Silverman, G.A., Little, R.D., Burke, D.T., Korsmeyer, S.J., Schlessinger, D. and Olson, M.V. (1989) Science 244,1348-1351.

45. Montanaro, V., Casamassimi, A., D'Urso, M., Yoon, J.-Y., Freije, W., Schlessinger, D., Muenke, M., Nussbaum, R.L., Saccone, S., Maugeri, S., Santoro, A.M., Motra, S. and Della Valle, G. (1991) Am. J. Hum Genet. 48, 183-194.

46. Riethman, H.C., Moyzis, R.K., Meyne, J., Burke, D.T., Otson, M.V. (1989) Proc. Natl. Acad. Sci. U.S.A. 86, 6240-6244.

47. Larin, Z., Monaco, A.P., Lehrach, H. (1991) Proc. Natl Acad. Sci. U.S.A. $88,4123-4127$.

48. Abidi, F.E., Wada, M., Little, R.D., Schlessinger, D. (1980) Genomics 7, 363-376. 